\title{
Repellent efficacy of DEET, MyggA, neem (Azedirachta indica) oil and chinaberry (Melia azedarach) oil against Anopheles arabiensis, the principal malaria vector in Ethiopia
}

\author{
Ephrem Abiy ${ }^{1 *}$, Teshome Gebre-Michael ${ }^{2}$, Meshesha Balkew ${ }^{2}$ and Girmay Medhin ${ }^{2}$
}

\begin{abstract}
Background: In Ethiopia, Anopheles arabiensis is the main vector responsible for the transmission of malaria in the country and its control mainly involves application of indoor residual spraying (IRS) and use of insecticide-treated bed nets (ITNs).

Objective: Although the role of repellents for reducing man-vector contact is documented in the literature, the response of An. arabiensis to repellents was not previously evaluated under field conditions in Ethiopia.

Method: The trial was conducted in Sodere village assessing the repellent activities of four repellents, of which, two of them were commercially available DEET ( $N$, N-diethyl-1,3-methylbenzamide) and MyggA (p-methane diol) and the other two were laboratory- produced, 20\% neem oil and 20\% chinaberry oil. A 6 by 6 Latin square design was employed by involving six volunteers who received rotated treatments of repellents and the Ethiopian Niger seed, noog abyssinia (Guizotia abyssinia), and locally called as noog oil (diluents to the two plant oils). Each volunteer also served as control. Volunteers were positioned at a distance of 20-40 m from each other and each was treated with one of the repellents, Niger seed/noog/ oil or untreated. Landing mosquitoes were collected from dusk to down using tests tubes. The tests were done in three replicates.

Results: Both DEET and MyggA provided more than 96\% protection. The mean protection time for DEET was 8 hrs while the time for MyggA was 6 hrs. Protection obtained from neem oil and chinaberry oil was almost similar (more than 70\%), however, the complete protection time for neem was 3 hrs, while that of chinaberry oil was one hour.
\end{abstract}

Conclusion: The commercial products and laboratory-produced repellents can be utilized by individuals to avoid contact with An. arabiensis in Ethiopia.

Keywords: DEET, MyggA, Chinaberry oil, Neem, Anopheles arabiensis, Vector, Repellent, Niger seed/noog/

\section{Background}

In Ethiopia, malaria is a leading cause of public health problem and impediment to socioeconomic development. Plasmodium falciparum and Plasmodium vivax are the two most common malaria parasites in Ethiopia, having annual percentage prevalence of $60 \%$ and $40 \%$, respectively [1-3].

\footnotetext{
* Correspondence: ephremabiy1@gmail.com

1The Carter Center Ethiopia, North Wollo Zone Health Department, Malaria and Trachoma project office, PO Box 69, Woldia, Northeast Amhara region, Ethiopia

Full list of author information is available at the end of the article
}

An important feature in the epidemiology of malaria in Ethiopia is the brevity of transmission season that precludes the development of immunity, favouring periodic epidemics with high mortality [2]. In general, knowing the biology, behaviour, habitat and identification of the vector species helps to decide and implement appropriate vector control methods [4]. Mosquitoes of the Anopheles gambiae complex are the most important malaria vectors in the world and are composed of seven sibling species namely, An. gambiae sensu stricto (s.s.), Anopheles arabiensis, Anopheles bwambae, Anopheles merus, Anopheles melas and Anopheles quadriannulatus 
(species A and B). However, only An. gambiae s.s. and An. arabiensis have become widely distributed and are most efficient vectors. Moreover, they are most important as far as intensified transmission of malaria is concerned [5,6]. Of the An. gambiae complex siblings, $A n$. arabiensis and An. quadriannulatus sp.B are known to occur in Ethiopia [7] and malaria in Ethiopia is transmitted mainly by $A n$. arabiensis $[8,9]$

Vector control is one of the measures applied to reduce malaria transmission by aiming at reducing breeding and survival of mosquito vectors. The available vector control methods are: chemical, biological, genetic, environmental management, personal protection and integrated vector management.

Although there are many vector control methods, most of them are too expensive, ecologically harmful, and environmentally unsafe or they are practically infeasible and inaccessible to be used in poor countries like Ethiopia. Moreover, insecticide resistance is now a major problem facing malaria vector control programnes in most African countries, including Ethiopia, with most important vector species, showing resistance to one or more of the insecticide classes used in vector control [4,8-10].

There is a need to have an intervention that better avoids such problems. This may include the uses of mosquito repellents which may be commercially available or locally produced by the community itself. Repellents have been used to drive away or repel insects or pests. They may be in the form of smoke, spray or aerosol, oils and body lotions. Aerosols and pump sprays are intended for skin applications and treating cloths while liquid, cream, lotion and spray products enable direct skin application. In Ethiopia, wogert (Silene macroserene), kebericho (Echinops kebericho), tinjut (Ostostegia integrifolia), and woira (Olea europaea) have been shown to have repellent effects against An. arabiensis under laboratory conditions [11]. Plant parts are burned indoors and the smoke is believed to repel and also knock down mosquitoes. They do have limitations in that they need to be applied frequently (hourly or daily) because of their short residual effect and they might have unpleasant side effects, such as coughing because of irritations from the smoke [12]. Therefore, it is important to know which repellent products can be relied on to provide predictable and prolonged protection from insect bites with out causing side effects on human health. So, rather than burning, repellents applied on the skin are preferable and comfortable for individual use. Moreover, insect repellents may be more economically feasible than other vector control methods and they can substitute or they can be alternatives to chemical control methods, such as space spray, which contaminate the environment and are less economically feasible [13].
Repellents are more recommended for people staying outdoors at night for work or leisure and those working in plantations and may be at risk during daytime. Repellents are also useful in combination with LLITNs by protecting people from the bite of mosquitoes before they retire to bed.

Therefore, a trial was conducted in Sodere village to evaluate the repellent efficacy of one synthetic repellent, DEET and three plant based repellents, Mygg A, 20\% neem oil and $20 \%$ chinaberry oil against An. arabiensis. This research project reported the results obtained from the trial.

\section{Methods}

Site of trial

The study was conducted in Sodere village situated in the Upper Rift Valley from February 2010 to March 2010. It is about $125 \mathrm{~km}$ east of Addis Ababa and $25 \mathrm{~km}$ south-east of Adama town (see Figure 1). Sodere is known for its recreational area because of its hot spring water for bath and swimming. The hot springs form ideal and abundant breeding sites for An. arabiensis throughout the year. The occurrence of An. arabiensis was previously reported [9].

\section{Test products}

The test materials were DEET (N, N-diethyl-1, 3methylbenzamide), MyggA, neem oil and chinaberry oil. DEET (a.i. 15\%; Peaceful-Sleep, Robertsons Home Care (Pty) Ltd., South Africa) was purchased locally from a supermarket. MyggA is a product from Sweden (19\% DEET with active perfume of terpene fraction of lavender, geranium and roses). Neem and chinaberry oils were previously hexane extracted at the Ethiopian Public Health Institute/EPHI/ [14] from seeds collected from middle Awash Valley. Edible oil/Niger seed oil/for diluents of neem and chinaberry oils was bought from a super market (See Figure 2).

\section{Repellent test procedure}

The testing methods are based on WHO guide lines [15] for repellent efficacy testing on human skin under field trials. Assessment was made by collecting mosquitoes which land on human volunteers whose legs were treated with repellents and controls. The dose of commercial repellents (DEET (N, N-diethyl-1, 3-methylbenzamide) and MyggA) was based on recommendations of the manufacturers. DEET (N, N-diethyl-1, 3-methylbenzamide) is said to be safe at a concentration of $22-35 \%$, but in the trial $15 \%$ was used as per the recommendation of the manufacturer. Neem and chinaberry oils were diluted to $20 \%$ using Niger seed (noog abyssinia) oil.

Six volunteers, all of them males with mean age of $22.1 \pm 6.9$ years, participated in the test. A 6 by 6 Latin- 


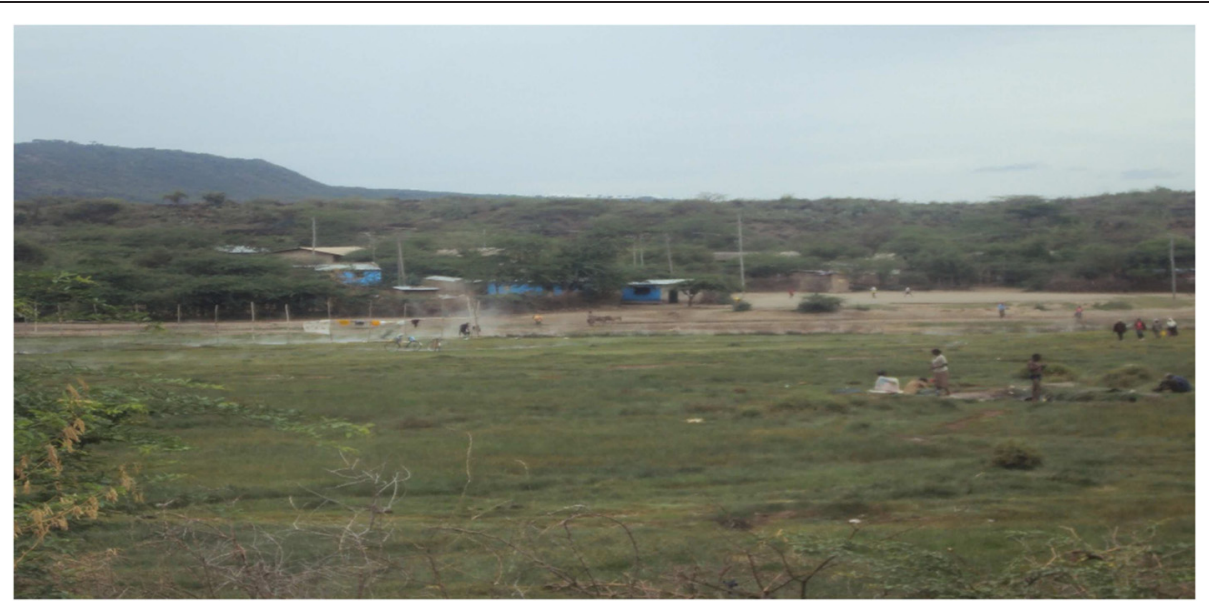

Figure 1 Part of Sodere village.
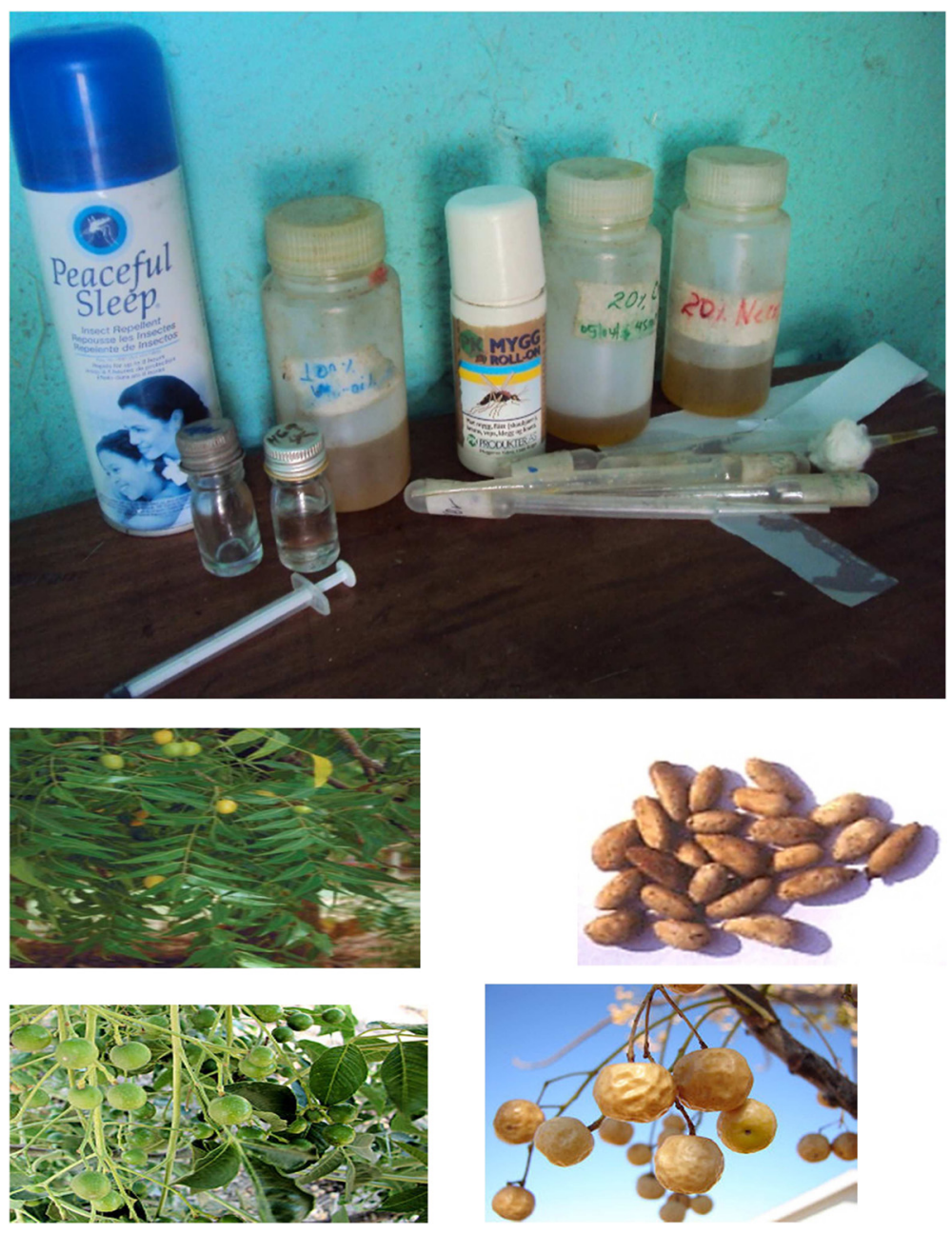

Figure $\mathbf{2}$ Repellents and equipment used in the test. 
square design was used for the tests. Thus, in the first night of the trial, a volunteer was treated with MyggA, and the second volunteer was treated with $20 \%$ neem oil, the third volunteer treated with edible oil, and the fourth volunteer with DEET, the fifth volunteer with $20 \%$ chinaberry oil and the sixth volunteer was control without the addition of repellent. On subsequent nights, the treatment application was rotated between the volunteers so that each volunteer was treated with different test repellents.

A volume of 2-3 $\mathrm{ml}$ of each of the repellents were applied on the bare legs of volunteers with syringes and spread evenly over the legs from the base of knee to the ankle. After the application of the repellents, volunteers were instructed not to rub, touch, or wet the treated legs (See Figure 3).

Every night volunteers were given test tubes labelled with the kind of treatment and time of mosquito collection. Volunteers were positioned at approximate distance of 20-40 metres from each other and landing mosquito collections took place from 7:00 pm to 6:00 am. A volunteer collected landing mosquitoes from his leg. Mosquitoes were identified in to distinct species based on morphological identification key of [16]. The trial was done in three replicates over a period of one month.

Volunteers were supplied with chloroquine $(250 \mathrm{mg})$ as chemoprophylaxis a week before the start, during and completion of the trial [15].

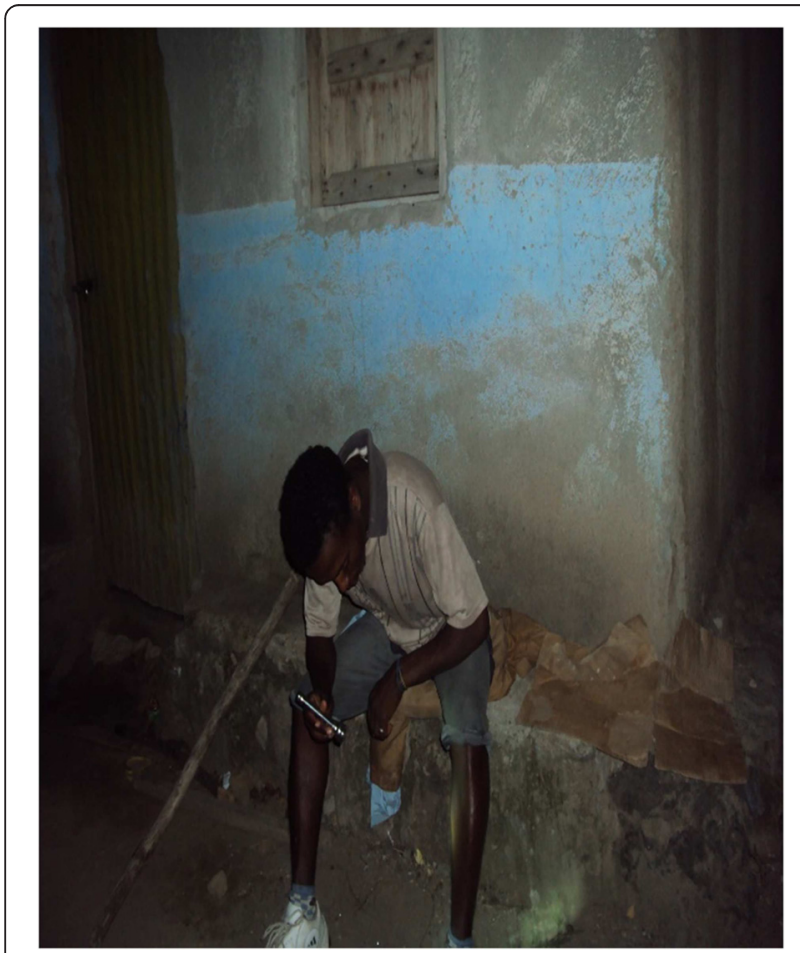

Figure $\mathbf{3}$ Volunteer collecting mosquitoes.

\section{Data analysis}

Comparison of repellency was made among the six treatment groups using SPSS-Version -13 and the number of $A n$. arabiensis collected was subjected to one-way analysis of variance (ANOVA) along with percent protection and complete protection time. Percentage Repellency was calculated as follows:

$$
\mathrm{P}=((\mathrm{C}-\mathrm{T}) / \mathrm{C}) \times 100 \%
$$

Where $T$ is the number of mosquitoes collected from treated volunteers $t$ and $C$ is the number of mosquitoes collected from untreated or positive control volunteers [15].

\section{Ethical considerations}

The study obtained ethical clearance from the Institutional Research Board (IRB) of ALIPB. Written consent of volunteers to participate in the trial was also obtained. Volunteers were given chloroquine as prophylaxis one week before the start of the study, every week during the trial and a week after the study was finalized. Treatment was given as per the treatment guideline, $250 \mathrm{mg}$ of $\mathrm{tab} / \mathrm{kg}$ of body weight (one tablet/week for four weeks).

\section{Results}

Overall, $857 \mathrm{An}$. arabiensis were collected, of which $0.5 \%$ were from volunteers treated with DEET, $1.8 \%$ from MyggA, 13.12\% from 20\% neem oil, 14.48\% from $20 \%$ chinaberry oil, $25.7 \%$ from control oil and $44.18 \%$ from control without oil (Table 1). Regarding the mean percentage protection, the commercial products performed much better than the laboratory prepared oils giving protection above $95 \%$ (Table 1). However, the percentage protection between DEET and MyggA is comparable $(\mathrm{p}=0.99)$. The mean percentage protection of $20 \%$ neem oil and $20 \%$ chinaberry oil is almost similar (p-value $=1.00$ ). Edible oil has also given $55 \%$ protection. The highest mean complete protection time was that of DEET ( $8 \mathrm{hrs})$ and the lowest was edible oil $(0 \mathrm{hr}$.). MyggA was able to score a mean complete protection time of 6 hrs.

Figure 4 depicts percent protection provided by repellents on hourly basis after treatment. The complete protection time of the commercial repellents was different, $8 \mathrm{hrs}$ for DEET and $6 \mathrm{hrs}$ for MyggA. For the first $6 \mathrm{hrs}$ both DEET and MyggA gave 100\% protection but after 2 hrs, protection of MyggA was decreased to $<80 \%$ while DEET gave $100 \%$. Neem provided $100 \%$ protection for $3 \mathrm{hrs}$ and then protection was dropped to $20-60 \%$ after 7 hrs. But, 20\% chinaberry gave $90-100 \%$ protection for the first $1 \mathrm{hr}$, and thereafter, dropped to $50-80 \%$ after 11 hrs. Although edible oil (control oil), showed percentage repellency of 55 (95\% CI $=45-65)$, the mean complete protection time was nil. Mosquito collection 
Table 1 Results of field evaluation of the repellent activities of MyggA, DEET, 20\% neem oil and $20 \%$ chinaberry oil against An. arabiensis in Sodere, Ethiopia

\begin{tabular}{|c|c|c|c|c|c|c|}
\hline Time (hr) & MyggA & DEET & neem oil & chinaberry oil & Edible oil & Control \\
\hline 19:00-20:00 & 0 & 0 & 0 & 1 & 2 & 15 \\
\hline 20:00-21:00 & 0 & 0 & 0 & 12 & 19 & 35 \\
\hline 21:00-22:00 & 0 & 0 & 1 & 16 & 27 & 53 \\
\hline 22:00-23:00 & 0 & 0 & 4 & 23 & 25 & 51 \\
\hline 23:00-24:00 & 0 & 0 & 30 & 27 & 37 & 57 \\
\hline $24: 00-1: 00$ & 0 & 0 & 29 & 14 & 51 & 60 \\
\hline 1:00-2:00 & 6 & 0 & 17 & 6 & 22 & 50 \\
\hline 2:00-3:00 & 5 & 0 & 9 & 6 & 12 & 22 \\
\hline $3: 00-4: 00$ & 4 & 2 & 15 & 10 & 10 & 30 \\
\hline 4:00-5:00 & 0 & 2 & 9 & 2 & 4 & 13 \\
\hline 5:00-6:00 & 0 & 0 & 1 & 0 & 1 & 10 \\
\hline Total & 15 & 4 & 115 & 117 & 210 & 396 \\
\hline \multicolumn{7}{|l|}{ MPP } \\
\hline$(95 \% \mathrm{Cl})$ & $96(92-100)$ & $98(96-100)$ & $71(58-84)$ & $70(63-77)$ & $55(45-65)$ & 0 \\
\hline MCPT & $6 \mathrm{hrs}$ & 8 hrs & 3 hrs & $1 \mathrm{hr}$ & 0 & 0 \\
\hline
\end{tabular}

MCPT $=$ Mean Complete Protection Time and MPP = Mean Percent Protection.

was low between 5:00 am and 6:00 am from all treatments including controls.

\section{Discussion}

The use of repellents as personal protective tools to break diseases transmission through avoidance of manvector contact is a usual way to combat vector-borne diseases [13]. Selection of appropriate repellents together with clothing and other personal protective tools can give adequate protection against bites of mosquitoes.

This study showed that both DEET and MyggA provided the highest comparable protection against $A n$. arabiensis and lowest protection from neem and chinaberry oil. Similar studies in Burkina Faso reported 95\% repellency and complete protection time of $8 \mathrm{hrs}$ against An. gambiae s.l. [17]. Similar observation was also made in Queensland, Australia on different mosquito species

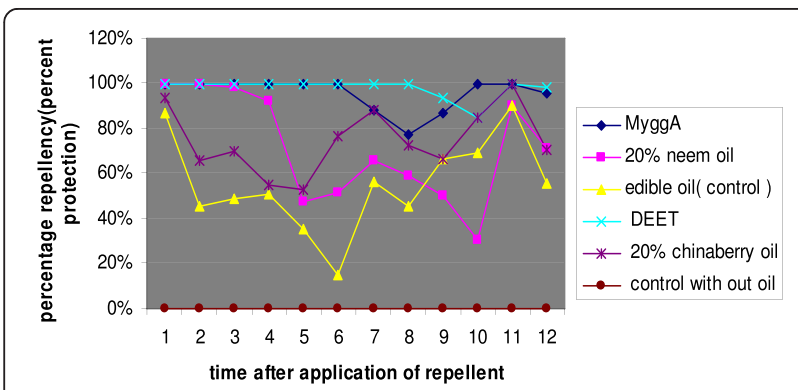

Figure 4 Percent protection provided hourly by Mugger, DEET, 20\% neem, 20\% chinaberry oil and 2 controls (control oil and control with out oil) against An. arabiensis in Sodere, Ethiopia.
[18]. However, a report from Thailand indicated lower repellency than stated above but the mean protection time was similar. This might be due to response variation between mosquito species and the concentration of the active ingredient [19], from Kenya a study has shown that DEET had given $60.2 \%$ protection against An. arabiensis after 9 hrs of treatment. Recent studies by $[20,21]$ from Australia and at Papua New Guinea showed that $20 \%$ DEET gave $>95 \%$ protection for only 2 hrs against all mosquitoes and overall of $55.8 \%$ against Culex at the end of 7 hrs of treatment.

In the present study, it was found that the complete protection time of $20 \%$ neem oil and $20 \%$ chinaberry oil is $3 \mathrm{hrs}$ and $1 \mathrm{hr}$, respectively in contrast to the report by [22], in which $2 \%$ neem provided 12 hrs protection from the bite of mosquitoes.

In India, 2\% neem oil diluted with 1-4\% coconut oil gave between $80 \%$ and $100 \%$ protection and four fold protection time than reported here [22-24]. The long protection may be due to effect of the combination neem with the diluents, coconut oil in the Indian test which differs from the present test that used edible oil. The other factor could be variation in the susceptibility status of Anopheles species in India (Anopheles stephensi, Anopheles sundaicus and Anopheles fluviatalis) and An. arabiensis.

This study has shown that MyggA provided 96\% (92-100\%) repellency and 6 hrs complete protection time against $A n$. arabiensis in Sodere. There are no documented reports to compare the results. The same is also true for chinaberry oil. 
Based on the results of this study, DEET was the most effective repellent against $A n$. arabiensis having a higher percent protection of $98 \%$ and complete protection time of $8 \mathrm{hr}(100 \%$ protection till $8 \mathrm{hr})$. As a result, it can be used and distributed in malaria-endemic areas or wherever there is a high vector capacity to prevent man-vector contact.

\section{Conclusions}

DEET was the most effective repellent against An. arabiensis because of its high percent repellency (98\%) and complete protection time $(8 \mathrm{hr})$. Mygg A also has shown a high potential of repellency (96\%) and protection time (6 hrs) next to DEET. $20 \%$ neem oil and 20\% chinaberry oil has shown less repellency (71\% and $70 \%$, respectively) and complete protection time ( $3 \mathrm{hrs}$ and $1 \mathrm{hr}$, respectively) as compared to DEET and MyggA.

\section{Recommendations}

The two commercially available repellents can be used and distributed to malaria endemic areas wherever there is a high biting rate of mosquitoes (high vectorial capacity). The two locally available oils can also be used, but to get several hours of protection applications can be repeated as much as required. In addition to ITNs these repellents can be used for people who work and rest out door.

\section{Competing interests}

The authors declare that they have no competing interests.

\section{Authors' contributions}

EA, TGM and MB designed the study, select the study area and carried out the investigation. GM edited the data analysis. EA wrote the manuscript. All authors read and approved the final manuscript.

\section{Acknowledgements}

All financial expenses for this project were funded by Aklilu Lemma Institute of Pathobiology, Addis Ababa University and Norwegian Programme for Development, Research and Education (NUFU). Communities in Sodere and volunteers involved are duly acknowledged.

\section{Author details}

${ }^{1}$ The Carter Center Ethiopia, North Wollo Zone Health Department, Malaria and Trachoma project office, PO Box 69, Woldia, Northeast Amhara region, Ethiopia. ${ }^{2}$ Aklilu Lemma Institute of Pathobiology, Addis Ababa University, PO Box 1176, Addis Ababa, Ethiopia.

Received: 19 December 2014 Accepted: 22 April 2015

Published online: 03 May 2015

\section{References}

1. MOH: Malaria prevention and control. Ministry of Health, Addis Ababa, Ethiopia, 2006. accessed from http://www.moh.gov.et/malaria.

2. $\mathrm{MOH}$. National five year strategic plan for malaria prevention and control in Ethiopia; 2006-2010. Addis Ababa, Ethiopia: Ministry of Health; 2007. p. 1-48.

3. $\mathrm{MOH}$. Entomological profile of malaria in Ethiopia. Addis Ababa, Ethiopia: Ministry of Health; 2009. p. 1-16.

4. Coetzee M. Distribution of the African malaria vectors of the Anopheles gambiae complex. Am J Trop Med Hyg. 2004;70:103-4.

5. White NJ. Malaria. In: Cook G, Zumla A, editors. Manson's Tropical Diseases, 21st edition. London: Elsevier Science Limited; 2003. p. 1205.
6. Service M. Medical entomology for students, Anopheline mosquitoes. 3rd edition. Cambridge University Press: Cambridge; 2004. p. 34-49.

7. Hunt RH, Coetzee M, Fetene M. The Anopheles gambiae complex: a new species from Ethiopia. Trans R Soc Trop Med Hyg. 1998;92:231-5.

8. Abose T, Yeebiyo Y, Olana D, Alamirew D, Beyene Y, Regassa L, et al. Re-orientation and definition of malaria vector control in Ethiopia. In: World Health Organization, WHO/MAL/98.1085. Geneva: WHO; 1998. p. 31.

9. Balkew M, Ibrahim M, Koekemoer LL, Brooke BD, Engers $H$, Aseffa A, et al. Insecticide resistance in Anopheles arabiensis (Diptera: Culicidae) from villages in central, northern and south west Ethiopia and detection of kdr mutation. Parasit Vectors. 2010;3:40.

10. Balkew M, Gebre-Michael T, Hailu A. Insecticide susceptibility level of Anopheles arabiensis in two agro-development localities in eastern Ethiopia. Parasitologia. 2003:45:1-3.

11. Karunamoorthi K, Adane M, Fentahun W. Laboratory evaluation of traditional insect/mosquito repellent plants against Anopheles arabiensis, the predominant malaria vector in Ethiopia. Parasitol Res. 2008;103:529-34.

12. Brain A, Smith L, Lines J, Ensink J, Cameron M. Smoke and malaria: are interventions to reduce exposure to indoor air pollution likely to increase exposure to mosquitoes? Trans R Soc Trop Med Hyg. 2007;101:1065-71.

13. Gupta RK, Rutledge LC. Laboratory evaluation of controlled-release repellent formulations on human volunteers under three climatic regimens. J Am Mosq Control Assoc. 1994;5:52-5.

14. Kebede Y, Gebre-Michael T, Blakew M. Laboratory and field evaluation of neem (Azedarchta indica A.Jus) and chinaberry (Melia azedarch L.) oils are repellents against Phlebotamus orientalis and P.bergroti (Diptera: Psychodidae) in Ethiopia. Acta Trop. 2009;113:145-50.

15. WHOPES. Guideline for efficacy testing of mosquito repellents for human skin. Control of neglected tropical diseases. WHO pesticide evaluation scheme (WHOPES). 2009:4:10-28.

16. Gillies MT, Coetzee M. A supplement to the Anophilinae of Africa south of the Sahara (Afro-tropical region). The South African Institute of Medical Research, Johannesburg, South Africa. 1987;55:127.

17. Costantinia C, Athanase B, Ilboudo-Sanogob E. Field evaluation of the efficacy and persistence of insect repellents DEET, IR3535 and KBR 3023 against Anopheles gambiae complex and other Afro tropical vector mosquitoes. Trans R Soc Trop Med Hyg. 2004;98:644-52.

18. Frances SP, Robert MM, Cassie CJ, Raethea LH, Robert DC. Laboratory and field evaluation of commercial repellent formulations against mosquitoes (Diptera: Culicidae) in Queensland, Australia. Australian J Entomol. 2005:44:431-6.

19. Walker TW, Robert LL, Coperland RA, Githeko AK, Wirtz RA, Githure JJ, et al. Field evaluation of arthropod repellents, deet and a piperdine compound, Al3-37220 against Anopheles arabiensis and Anopheles funestus in western Kenya. J Am Mosq Control Assoc. 1996;2:172-6.

20. Frances SP, Mackenzie DO, Klun JA, Debboun M. Laboratory and field evaluation of SS220 and DEET against mosquitoes in Queensland, Australia. J Am Mosq Cont Ass. 2009;25:174-5.

21. Frances SP, Cooper RD, Popat S, Sweeny AW. Field evaluation of the repellents, DEET, CLC4, and Al3-37220 against Anopheles in Lae, Papua New Guinea. J Am Mosq Control Assoc. 1999;15:339-41.

22. Sharma VP, Ansari MA, Razdan RK. Mosquito repellent action of neem (Azadirachta indica) oil. J Am Mosq Cont Assoc. 1995;9:359-60.

23. Sharma VP, Nagpal BN, Srivastava A. Effectiveness of neem oil mats in repelling mosquitoes. Trans R Soc Trop Med Hyg. 1993;87:627-8.

24. Mishra AK, Singh N, Sharma VP. Use of neem oil as a mosquito repellent in tribal villages of Mandla district, Madhya Pradesh. Indian J Malariol. 1995;32:99-103. 